\title{
Age-Dependent Aggregation of a-Synuclein in the Nervous System of Gut-Brain Axis is Associated with Caspase-1 Activation
}

Qi Hu

Tongji Hospital of Tongji Medical College of Huazhong University of Science and Technology Mei Hong

Yangtze University

\section{Mengyang Huang}

Central Hospital of Wuhan: The Central Hospital of Wuhan

\section{Quan Gong}

Yangtze University

\section{Xiaofan Zhang}

Tongji Hospital of Tongji Medical College of Huazhong University of Science and Technology https://orcid.org/0000-0003-1025-164X

\section{Vladimir N. Uversky}

USF Health Byrd Alzheimer's Institute

\section{Francisco Pan-Montojo}

Ludwigsburg Hospital: Klinikum Ludwigsburg

\section{Teng Huang ( $\nabla$ teahuangteng@126.com )}

Tongji Hospital of Tongji Medical College of Huazhong University of Science and Technology Honglian Zhou

Tongji Hospital of Tongji Medical College of Huazhong University of Science and Technology Suiqiang Zhu

Tongji Hospital of Tongji Medical College of Huazhong University of Science and Technology

\section{Research Article}

Keywords: a-Synuclein, Caspase-1, Rotenone, Aging, Parkinson' desease

Posted Date: December 6th, 2021

DOI: https://doi.org/10.21203/rs.3.rs-1095808/v1

License: (c) (1) This work is licensed under a Creative Commons Attribution 4.0 International License. Read Full License 
Version of Record: A version of this preprint was published at Metabolic Brain Disease on January 28th, 2022. See the published version at https://doi.org/10.1007/s11011-022-00917-6. 


\section{Abstract}

a-Synuclein (a-Syn) plays a key role in the development of Parkinson' desease (PD). As aging is acknowledged to be the greatest risk factor for PD, here we investigated a-Syn expression in the ileum, thoracic spinal cord, and midbrain of young (1-month-old), middle-aged (6-, 12-month-old) to old (18month-old) mice. We demonstrated that both the levels of a-Syn monomers, oligomers and ratios of oligomers to monomers were increased with aging in the ileum, thoracic spinal cord, and midbrain. Whereas, the expression of tyrosine hydroxylase (TH), the rate-limiting enzyme for dopamine synthesis, was decreased with aging in the midbrain. We failed to find corresponding a-Syn mRNA increase with aging. However, we found an increased expression of caspase-1 in the ileum, thoracic spinal cord, and midbrain. A specific caspase-1 inhibitor VX765 significantly reduced levels of both the a-Syn monomers and oligomers triggered by the rotenone in vitro. Taken together, the increase in a-Syn aggregation with aging might not occur first in the gut, but simultaneously in the nervous system of gut-brain axis.. The mechanism of the age-dependent aggregation of a-Syn in nervous system is likely triggered by the agingrelated caspase-1 activation.

\section{Introduction}

Parkinson's disease (PD) is a neurodegenerative disorder, which is characterized by the death of dopaminergic neurons in the substantia nigra (SN) and the presence of Lewy bodies (LBs) and Lewy neurites (LNs) as cytosolic inclusions in surviving neurons. It is suggested that a-Synuclein (a-Syn) is the major component of LBs. In addition, a-Syn is associated with the pathogenesis of PD(Goedert, 2001), as several mutations associated with the early onset of PD are found in this protein (e.g. A30P(Kruger et al., 1998), E46K(Zarranz et al., 2004) and A53T(Polymeropoulos et al., 1997), which are known to the scientific community for relatively long time, and several more recently discovered $\mathrm{H} 50 \mathrm{Q}$ (Appel-Cresswell et al., 2013; Proukakis et al., 2013), G51D(Kiely et al., 2013; Lesage et al., 2013), and A53E(Pasanen et al., 2014) mutants). a-Syn is a widespread neuronal presynaptic protein located in the nervous system, and with lower expression in blood cells, pancreas, heart, and skeletal muscle(Brighina et al., 2010; Ltic et al., 2004). Accumulated evidence suggests that a-Syn does not have a unique biological function, but instead it serves as an illustrative example of multifunctional intrinsically disordered protein(Breydo et al., 2012; Silva et al., 2013; Uversky, 2017). Given that the abundance of a-Syn in the presynaptic nerve terminals and its ability to bind to lipid membranes, this protein may play a critical role in in neural transmission(Davidson et al., 1998), the release of neurotransmitters, neuronal plasticity, learning and development(Krainc et al., 2017). a-Syn is prone to form aggregates when present at high levels. Furthermore, a-Syn is a heat-stable soluble protein binding several other proteins, which can promote conformational changes of physiological relevance or lead to abnormal aggregation as well(Breydo et al., 2012; Silva et al., 2013; Uversky, 2017).

Several genetic causes and multiple environmental factors, such as pesticides, can affect the misfolding and aggregation of a-Syn. The aging also plays an important role in regulating the expression and function of a-Syn. Some investigations revealed that age-dependent decreases in a-Syn were widespread 
throughout the brain, affecting other regions (e.g., hippocampus) besides the SN both in rats(Adamczyk et al., 2005) and mice(Mak et al., 2009b). On the contrary, more investigations showed that a-Syn was increased with aging. Chu et al. reported that a-Syn within individual nigral neurons was increased with aging both in non-human primates and in humans, and the aggregation of a-Syn was associated with the decreases in tyrosine hydroxylase (TH) with aging(Chu and Kordower, 2007). Canron et al. found that age-dependent a-Syn aggregation occurred in mouse lemur primates, and a-Syn aggregates in the cytoplasm were observed only in aged animals in different brain regions(Canron et al., 2012). In the homozygous A53T mice, striatal A53T a-Syn expression was increased with aging. a-Syn expression was significantly elevated in the 12 month-old A53T mice, when compared with the 2 month-old controls(Oaks et al., 2013). It has also been reported that the expression and accumulation of a-Syn were increased gradually with aging in neurons of Meissner's and Auerbach's plexuses of the gastrointestinal tract(Zhong et al., 2017).

Physiologically, a-Syn expression is restricted to the presynaptic terminals in most neural systems(Halliday and McCann, 2008). In contrast, age-dependent increase in a-Syn appears regularly in axonal swellings and distorted terminal fields within the myenteric ganglia, as well as within the nonganglionic regions adjacent to the ganglia throughout the gastrointestinal tract(Phillips et al., 2009). Interestingly, there is growing evidences suggesting that the intestines are affected in PD(Houser and Tansey, 2017) as well. Indeed, the incidences of the debilitating digestive symptoms may be partly explained by the presence of inclusions and debris immunoreactive for a-Syn in the enteric nervous system (ENS)(Shprecher and Derkinderen, 2012). Furthermore, constipation and anosmia both appear in early PD. Consistently, synucleinopathy affects the colonic nerves, olfactory bulb, and the cardiac sympathetic system at first(Orimo et al., 2008). Therefore, it is speculated that synucleinopathy may spread from the gastrointestinal tract or the olfactory bulb to the brain via the prion-like mechanisms(Frost and Diamond, 2010; Holmqvist et al., 2014a).

Based on these observations, it was reasonable to assume that a-Syn in the nervous system of gut may be susceptible to pathogens of infection, thereby converting healthy aging into pathological aging. Being an intrinsically disordered protein, a-Syn is known to exist in several conformational and multimeric/oligomeric forms under physiological conditions. Changes in a-Syn protein levels, conformational and (mis)folding state indicate the transition towards pathological conditions. Furthermore, synucleinopathy triggered in ENS may progress from the gastrointestinal tract to the brain and lead to develop classic PD motor symptoms. Therefore, it is critical to characterize whether a-Syn aggregates occur simultaneously in the nervous system of gut-brain axis, or emerged firstly in the gut with healthy aging. Innate immune system can be activated during aging and neurodegeneration. Inflammasomes, which acted as an important component of innate immune system, lead an aggregation of aSyn in neuronal cell culture(Heneka et al., 2018). This aggregation is caused by truncation of aSyn by caspase-1, an enzyme component of the inflammasome(Wang et al., 2016). Caspase-1 activated by inflammation can shift the physiological monomer-multimer equilibrium in the direction of the more protease-sensitive monomeric forms of a-Syn, thereby promoting formation of potentially cytotoxic oligomers and ultimately causing neuronal dysfunction and loss(Nuber and Selkoe, 2016). The aims of 
the present study were to investigate the changes in the aggregation levels of a-Syn in the gut, spinal cord, and midbrain with aging, and to assess the potential role of caspase- 1 in the process of a-Syn aggregation.

\section{Methods And Materials}

\section{Animals}

One-month old male C57BL/ 6 mice and one-year old male C57BL/ 6 mice were purchased from from the Hubei Provincial Center for Disease Control and Prevention (Wuhan, China). The animals were housed under standard animal care conditions and had free access to water and chow ad libitum. The mice were sacrificed on the 1, 6, 12 and 18-month respectively. All procedures carried out according to the ethical guidelines of the Animal Care and Use Committee of Huazhong University of Science and Technology.

\section{Cell culture}

The human dopaminergic neuroblastoma (SH-SY5Y) cells were obtained from the Type Culture Collection of the Chinese Academy of Sciences (Shanghai, China), and were grown in Dulbecco's modified Eagle medium (DMEM)/F-12 medium (Nacalai Tesque, Kyoto, Japan) supplemented with $10 \%$ fetal bovine serum (Life Technologies, Carlsbad, CA, USA), $50 \mathrm{U} / \mathrm{ml}$ penicillin (Nacalai Tesque, Kyoto, Japan), and 50 $\mu \mathrm{g} / \mathrm{mL}$ streptomycin (Nacalai Tesque, Kyoto, Japan) under a humidified 5\% CO2-enriched at atmosphere at $37^{\circ} \mathrm{C}$. Subsequently, the SH-SY5Y cells were assigned into 4 groups: $100 \mathrm{nM}$ rotenone group, $200 \mathrm{nM}$ rotenone group, VX765 group and control group. In the $100 \mathrm{nM}$ and $200 \mathrm{nM}$ rotenone group, SH-SY5Y cells treated with rotenone $100 \mathrm{nM}$ and $200 \mathrm{nM}$ for $24 \mathrm{~h}$. In VX765 group, SH-SY5Y cells were pretreated $50 \mu \mathrm{M}$ caspase-1 specific inhibitor VX765 and 200nM rotenone were added to the culture medium subsequently. In the control group, SH-SY5Y cells were just cultured with control solution. After incubation at $37^{\circ} \mathrm{C}$ in a humidified $5 \%$ (vol/vol) $\mathrm{CO} 2$ incubator overnight, the cells were harvested for immunofluorescence array and Western blotting.

\section{Immunofluorescence}

Immunofluorescence was perforemd as previously described. Mice were sacrificed and perfused transcardially with $4 \%$ paraformaldehyde (PFA). The ileum, thoracic spinal cord and midbrain were harvested and fixed in 4\% PFA, serial 10-mm-thick slices prepared. The following primary antibodies were used: rabbit anti-a-Syn (1:400, Santa Cruz Biotechnology, Santa Cruz, CA), sheep anti-Tyrosine Hydroxylase (TH) (1:800, Abcam, Cambridge, UK), and 33 -Tubulin (TU-20) mouse mAb (1:200, Cell Signaling Technology CST, Boston, MA). Expression visualized by the FITC-conjugated goat-anti-rabbit IgG, Cy3conjugated goat-anti-mouse IgG, and Cy3-conjugated rabbit-anti-sheep IgG (Millipore). The stain was evaluated using a Zeiss confocal microscope (LSM 710, Carl Zeiss Jena, Germany), and the intensity of a-Syn expression in the SN pars reticulate, as well as TH-positive cells in SN was measured using the Image $\mathrm{J}$ software (National Institute of Health, Bethesda, MA). 


\section{Western blotting analysis}

Western blotting analysis was conducted as previously described. Briefly, twenty micrograms of denatured protein were loaded per lane and separated on $12 \%$ gels by sodium dodecyl sulfatepolyacrylamide gel electrophoresis (SDS-PAGE). After electrophoresis, separated proteins were blotted to polyvinyl difluoride membranes (GE Healthcare, Buckinghamshire, UK). For detection of a-Syn and GAPDH, the following antibodies were used after blocking of the membranes: rabbit anti-a-Syn and rabbit anti-caspase-1 (1:200, Santa Cruz Biotechnology), and rabbit anti-glyceraldehyde-3-phosphate dehydrogenase (GAPDH) (1:20000, Sigma-Aldrich). Incubation with primary antibodies was carried out overnight at $4^{\circ} \mathrm{C}$ with gentle shaking overnight. Then, a secondary goat anti-rabbit IgG (1:5000, Santa Cruz Biotechnology) used to recognize the primary antibody. Membranes were developed with ECL Western blotting substrate (GE Healthcare). Quantification of band density was performed using Image $\mathrm{J}$ software.

\section{Real-time PCR}

a-Syn mRNA expression was examined by Real-time PCR. Briefly, total RNA was isolated from tissue samples using the RNeasy kit (Qiagen, Hilden, Germany). The cDNA synthesis was performed using the First-Strand cDNA synthesis kit (Invitrogen, Carlsbad, CA). Real-time PCR analyses were performed on an Mx3000P QPCR System (Stratagene, La Jolla, CA) using Fast SYBR Green Master Mix (Toyobo, Osaka, Japan) and primers for amplification of a-Syn (sense, 5'-GATC CTGGCAGTGAGGCTTA-3', antisense, 5'GCTTCAGGCTCATAGTCTTGG-3'), and $\beta$-actin (sense, 5'-AGAGGGAAATCGTGCGTGAC-3', antisense, 5'CAATAGTGATGACCTGGCCGT-3'). Relative quantification of target mRNA expression was calculated and further normalized to $\beta$-actin.

\section{Statistical analysis}

The data were expressed as the means \pm standard deviation (SD). Data were compared using unpaired, two-tailed Student's t-test. All tests were performed by IBM SPSS25.0. A p-value below 0.05 was considered indicating statistical significance.

\section{Results}

\section{Age-dependent increase of a-Syn immunoreactivity in the ileum}

As growing evidence suggesting that PD also affects the gut and the incidences of the debilitating digestive symptoms may partly be explained by the presence of inclusions and debris immunoreactive for a-Syn in the enteric nervous system [26], the a-Syn accumulation was evaluated in the enteric nervous system with aging and immunofluorescence analysis of samples from the 1-, 6-, 12-, and 18-month-old mice was performed. As shown in Figure 1, the amount of a-Syn immunoreactivity in the ileum was systematically increased with aging. Furthermore, there was a significant difference in the levels of ileum 
a-Syn immunoreactivity in samples from the 18-month-old group compared to the 1-monthold group $(p<0.05$, Figure 1$)$.

\section{Age-dependent increase of a-Syn immunoreactivity in the thoracic spinal cord}

In order to investigate whether a-Syn immunoreactivitychanges in the thoracic spinal cord with aging, immunofluorescence analysis of samples from the 1-, 6-, 12-, and 18-month-old mice was performed. The analysis showed that the levels of a-Syn immunoreactivity in the thoracic spinal cord increased with aging. There was a significant difference in levels of the thoracic spinal cord a-Syn immunoreactivity from samples of 18-month-old group compared to samples from the 1-monthold group ( $p<0.05$, Figure 2). Furthermore, predominant location of a-Syn immunoreactivity in the thoracic spinal cord was changed with aging. In the 1-month- and 6-month-old groups, the a-Syn immunoreactivity was predominantly presented in white matter of the thoracic spinal cord. However, the a-Syn immunoreactivity was predominantly displayed in grey matter of the thoracic spinal cord in the 12month- and 18-month-old group.

\section{Age-dependent increase of a-Syn immunoreactivity in the midbrain}

To investigate variation of the a-Syn immunoreactivity in the midbrain with aging, immunofluorescence was performed using samples from at 1-, 6-, 12-, and 18-month-old mice. This analyses revealed that the levels of a-Syn immunoreactivity in the midbrain were increased with aging, while there was a trend of age-related decreases in the number of dopminegic neuron. There was a significant difference in the levels of the midbrain a-Syn immunoreactivity from 18-month-old group compared to the those of the 1month-old group ( $p<0.05$, Figure 3 ). However, there was no significant difference in the number of dopaminergic neuron among all groups.

\section{Age-dependent increase in the levels of a-Syn monomers and oligomers in the ileum, the thoracic spinal cord, and the midbrain}

To examine whether the levels of a-Syn aggregation are changed with aging in vivo, a-Syn monomers and oligomers in the ileum, thoracic spinal cord, and midbrain were detected by Western blotting in samples from at 1-, 6-, 12-, and 18-month-old mice. This analysis revealed that the levels of a-Syn monomers and oligomers in the ileum, thoracic spinal cord, and midbrain were increased with aging (Figure 4A). The levels of a-Syn monomers in the ileum, thoracic spinal cord, and midbrain obtained from 18-month-old group were significantly higher than those in the corresponding tissues from the 1-month-old group $(p<0.05$, Figure 4B). Meanwhile, there was no significant difference between the 1-month-old group and the 12-month-old group. Besides, the levels of a-Syn oligomers in the ileum, thoracic spinal cord, and midbrain obtained from the 18-month-old group were also significantly increased in the corresponding tissues when compared to those form the 1-month-old group. Interestingly, the significant increase was also detected at the 12-month in the thoracic spinal cord and midbrain as well ( $p<0.05$, Fig $4 C$ ). Furthermore, the ratios of oligomers to monomers in every group was analyzed. The results showed that the ratios of oligomers to monomers in the ileum, thoracic spinal cord, and midbrain obtained from 18- 
month-old group were all significantly higher than obtained from the 1-month-old group ( $p<0.05$, Fig 4D). Moreover, the ratios in the ileum and midbrain from the 12-month-old group were increased significantly when compared with the 1-month-old group ( $p<0.05$, Fig 4D).

\section{Age-dependent decrease in the a-Syn mRNA expression levels in the ileum, thoracic spinal cord, and midbrain}

To further confirmed changes in the expression of a-Syn with aging, the levels of $a$-Syn mRNA were quantified at $1,6,12$, and 18 month in the ileum, thoracic spinal cord, and midbrain respectively. As shown in Figure 5, there was no significant change in the levels of a-Syn mRNA with age among all groups, although $a$-Syn mRNA expression decreased gradually with aging (Figure 5).

\section{Simultaneous age-dependent increase in the caspase-1 levels in the ileum, thoracic spinal cord, and midbrain}

Cellular stress triggers caspase-1 activation and caspase-1-mediated release of interleukin-1 (IL-1) and IL18 , which triggers an inflammatory response. In neurodegenerative diseases, the continued accumulation of misfolded protein aggregates can trigger and maintain the activation of inflammasome, which drives CNS inflammation and neuropathology(Gordon et al., 2018). Previous studies have suggested the possibility of activated caspase-1 directly cleaving a-Syn into smaller fragments(Wang et al., 2016). To test whether the levels of caspase- 1 vary with aging as well, caspase-1 expression in the ileum, thoracic spinal cord, and midbrain at 1, 6, 12, and 18 months was quantified by Western blotting analysis. This analysis indicated that the levels of caspase- 1 increased gradually with the age. Furthermore, the leveles of caspase-1 in the 18-month-old group were significantly higher than those in the 1-month-old group $(p<0.05$, Figure 6).

\section{Inhibition of caspase-1 activation induces decrease in the a-Syn accumulation}

To see whether caspase- 1 is involved in the a-Syn accumulation, we treated SH-SY5Y cells with the caspase-1-specific inhibitor VX765 and monitored its effects on the aggregation of a-Syn. Western blot analysis showed that both $100 \mathrm{nM}$ and $200 \mathrm{nM}$ rotenone treatment increased the levels of a-Syn monomers and oligomers in the SH-SY5Y cells. Furthermore, the levels of a-Syn monomers and oligomers in $200 \mathrm{nM}$ rotenone group were significantly higher than in the control group. However, VX765 significantly reduced the levels of both oligomers and monomers of a-Syn in $200 \mathrm{nM}$ rotenone group $(p<0.05$, Figure 7). Futhermore, immunofluorescence staining also demonstrated that caspase- 1 inhibitor VX765 could inhibit the a-Syn accumulation promoted by rotenone (Figure 8).

\section{Discussion}

The current study was designed to evaluate age-related changes in the a-Syn expression in mice. The results of Western blot and immunofluorescence revealed that aging was associated with the simultaneous elevation of the levels of a-Syn monomers and oligomers in the ileum, the thoracic spinal 
cord, and the midbrain. Furthermore, the expression of caspase-1 was also increased in all the above tissues with aging. Inhibition of caspase-1 could decrease levels of the rotenone-triggered a-Syn accumulation in SH-SY5Y cells. Collectively, these findings indicate that age-dependent accumulation of a-Syn in the nervous system may be associated with caspase- 1 activation with aging .

PD is a common neurodegenerative movement disorder. One of pathological hallmarks of PD is the accumulation and aggregated a-Syn in the cytosol as LBs and as LNs in the axons. Based on the epidemiological studies, aging is acknowledged to be the greatest risk factor for the PD development(Bennett et al., 1996; Morens et al., 1996). Interestingly, our results demonstrated that the levels of a-Syn monomers and oligomers were gradually elevated with aging. Furthermore, the levels of aSyn monomers and oligomers in the 18-month-old group were significantly higher than in the 1-month-old group. Meanwhile, accumulating evidences suggest that oligomeric forms of a-Syn are the most neurotoxic species, which are linked to the development of neuron loss and sporadic PD. It is reasonable to speculate that aging-related elevation in the levels of a-Syn oligomers can lead to an increased risk for the development of sporadic PD through neurotoxicity of oligomers. Simultaneously, our results showed that the ratio of oligomers to monomers in the ileum, thoracic spinal cord, and midbrain obtained from 18-month-old group were significantly higher than those from the 1-month-old group. The elevated ratios of oligomers to monomers may cause that a-Syn is more susceptible to external aggressors and develop more aggregates during aging. It is likely that a-Syn aggregation may induce transient or bland symptoms that may occur in healthy people for many years or even decades before the actual PD onset.

We demonstrated that a-Syn was accumulating in neurons in an age-dependent manner, and was assossated with a robust down-regulation of $\mathrm{TH}$, one of the earliest cellular manifestations in the $\mathrm{SN}$ degeneration in PD(Ross et al., 2004). Such age-related decrease in the TH may lead to the striatal dopamine loss, which is one of the first degenerative events in the vulnerable dopaminergic neurons. These findings suggest that a-Syn aggregation may be a trigger for early pathological cascade. Several studies have reported the age-related loss of dopaminergic neurons(Rudow et al., 2008). The decreased $\mathrm{TH}$ in current study is in agreement with the previous reports showing that TH was reduced with aging, especially after the middle age(Chu et al., 2002). The present study examined the expression of a-Syn associated with healthy aging in mice and found a profound age-related increase in the levels of both aSyn monomers and oligomers, which was associated with the age-related TH decrease. However, it remains unclear whether the physiological aging-related overexpression of a-Syn could cause the reduction of dopamine phenotype. If a-Syn overexpression is related to the reduction of dopamine phenotype, then inhibition of the expression of this protein may reverse the loss of striatal dopamine and alleviate cardinal symptoms. It is worth noting that age-related changes in a-Syn mRNA, with a systemating reduction occurring in a transiton from young to middle-aged, and to old age in mice. These findings were different from other observations, which showed relatively paralleled changes of a-Syn protein with a loss of mRNA in the SN(Mak et al., 2009a). After peaking at early postnatal days, a-Syn mRNA expression levels decline in the null mutant rodent brain throughout young, mature, and old age $(\mathrm{Li}$ et al., 2004). This decrease might be counteracted by posttranslational mechanisms of protein stabilization that allows a-Syn levels to remain relatively high and stable(Li et al., 2004). Hence, the 
elevated levels of a-Syn monomers and oligomers should not be caused by the elevated levels of the aSyn mRNA and the overpression of this protein.

It has been reported that PD-linked missense mutations can increase production of the C-terminally truncated a-Syn species(Li et al., 2005), which can accelerate aggregation of the full-length a-Syn protein both in vitro(Li et al., 2004) and in vivo(Ulusoy et al., 2010), and thereby may contribute to or even initiate the aggregation process. It was reported that caspase- 1 can directly cleave a-Syn and generate Cterminally truncated forms of a-Syn, which may initiate or accelerate a-Syn aggregation(Bassil et al., 2016; Wang et al., 2016). Our results also demonstrated that the treatment of neurons with the caspase-1 inhibitor VX-765 decrease levels of both a-Syn monomers and oligomers triggered by the rotenone. We also found an increased expression of caspase- 1 in the ileum, thoracic spinal cord, and midbrain with aging. It is well known that the levels of reactive oxygen species (ROS) are increased with aging. ROS is one of the crucial elements for the pyrin domain-containing 3 (NLRP3) inflammasome activation. NLRP3 inflammasome can activate caspase-1, leading to the initiation of the a-Syn aggregation process(Tschopp and Schroder, 2010). Therefore, it is possible that caspase-1 may be activated by oxidative stress with aging and may be associated with the age-dependent increase in the a-Syn accumulation in nervous system.

Given that enteric neurons independently control the secretion and movement of the gastrointestinal tract, the ENS is known as the 'second brain'(Avetisyan et al., 2015). The ENS plays an important role in the PD onset(Hawkes et al., 2009). a-Syn is widely expressed in the autonomic nerves that dominate the gastrointestinal tract(Pouclet et al., 2012). Dense aggregates of a-Syn progressively accumulate in the intrinsic and extrinsic elements of the ENS with aging(Phillips et al., 2009). Indeed, the presence of inclusions and debris immunoreactive for a-Syn in the ENS may partially explain the incidences of debilitating digestive symptoms(Pouclet et al., 2012) and age-related degenerative processes, such as PD, in elderly individuals(Shprecher and Derkinderen, 2012). In the gastrointestinal tract, pathogens or exposure to other factors may trigger a-Syn aggregation through axons of the myenteric (Auerbach's) plexus, as well as via postganglionic neurones of the submucosal (Meissner's) plexus(Gelpi et al., 2014) after entering the nervous system. At the early stages of PD, increased intestinal permeability is associated with the presence of a-Syn in the intestinal mucosa(Forsyth et al., 2011). Therefore, the mucosa tract could be a potential starting point for generation of aggregated a-Syn species in terms of the impact of environmental risk factor, and the vagus nerve can act as a "highway", by which pathology may be transmitted to the lower brain(Braak et al., 2003; Holmqvist et al., 2014b). However, does the aSyn aggregation emerge first in the gastrointestinal tract in terms of the impact of aging as well? Interestingly, our study revealed that the increased a-Syn levels, the elevated ratio of oligomers to monomers and the presence of a-Syn aggregates did not happen firstly in the gut, but emerged simultaneously in the nervous system of gut-brain axis. These results therefore indicate that the early onset PD might have different pathological pattern from the late onset PD. Although there is much evidence to support Braak's hypothesis that Lewy pathology spreads from the peripheral nervous system to the central nervous system, our observations support the speculation that Braak staging might not be uniquely applicable for various subtypes of PD(Jellinger, 2019). 
However, we recognize that our study has several limitations. First, the behavioural information to support the aging-related a-Syn changes is lacking. Second, we did not test the effects of VX-765 treatment on the endogenous concentrations of dopamine neurotransmitters, which might provide important information on the caspase- 1 effects on aging. Third, the mechanisms by chich caspase- 1 is activated via oxidative stress with aging still need to be thoroughly investigated.

\section{Conclusion}

In conclusion, our data showed that the increased a-Syn with aging did not started firstly in the gut, but emerged simultaneously in the ileum, spinal cord, and midbrain. The age-dependent increase in the a-Syn accumulation in nervous system is likely related to the caspase- 1 activation. These findings suggest that the levels of the a-Syn protein may increase with aging, and these age-related increase may be associated with decrease in the nigrostriatal activity. These findings could provide information for the classification of PD subtypes.

\section{Declarations}

Funding This work was supported by grants from the National Natural Science Fund of China (NSFC) (81102689) and Fangu Foundation.

Conflict of interest The authors declare that there is no conflict of interest.

Author contributions Q.H, M.H. and M.H. performed experiments and analyzed the data, Q.G. and X.Z. designed the study, V.N.U., F.PM., H.Z. and S.Z assisted with the animal experiments. Q.H. and T.H. wrote the manuscript. All authors revised and approved the final version of the manuscript.

Data availability Data included in article/supplementary material/referenced in article.

Ethical approval All animal procedures were approved by the Tongji Hospital animal Care and Use Committee in line with the USA National Institutes of Health $(\mathrm{NIH})$ guidelines.

\section{References}

1. Adamczyk, A., Solecka, J. and Strosznajder, J.B., 2005. Expression of alpha-synuclein in different brain parts of adult and aged rats. J Physiol Pharmacol, 56(1): 29-37.

2. Appel-Cresswell, S. et al., 2013. Alpha-synuclein p.H50Q, a novel pathogenic mutation for Parkinson's disease. Mov Disord, 28(6): 811-3. https://doi.org/10.1002/mds.25421.

3. Avetisyan, M., Schill, E.M. and Heuckeroth, R.O., 2015. Building a second brain in the bowel. J Clin Invest, 125(3): 899-907. https://doi.org/10.1172/jci76307.

4. Bassil, F. et al., 2016. Reducing C-terminal truncation mitigates synucleinopathy and neurodegeneration in a transgenic model of multiple system atrophy. Proceedings of the National 
Academy of Sciences of the United States of America, 113(34): 9593-8. https://doi.org/10.1073/pnas.1609291113.

5. Bennett, D.A. et al., 1996. Prevalence of parkinsonian signs and associated mortality in a community population of older people. The New England journal of medicine, 334(2): 71-6. https://doi.org/10.1056/NEJM199601113340202.

6. Braak, H. et al., 2003. Staging of brain pathology related to sporadic Parkinson's disease. Neurobiology of aging, 24(2): 197-211. https://doi.org/10.1016/s0197-4580(02)00065-9.

7. Breydo, L., Wu, J.W. and Uversky, V.N., 2012. Alpha-synuclein misfolding and Parkinson's disease. Biochim Biophys Acta, 1822(2): 261-85. https://doi.org/10.1016/j.bbadis.2011.10.002.

8. Brighina, L. et al., 2010. Lymphomonocyte alpha-synuclein levels in aging and in Parkinson disease. Neurobiology of aging, 31(5): 884-5. https://doi.org/10.1016/j.neurobiolaging.2008.06.010.

9. Canron, M.H., Perret, M., Vital, A., Bezard, E. and Dehay, B., 2012. Age-dependent alpha-synuclein aggregation in the Microcebus murinus lemur primate. Scientific reports, 2: 910. https://doi.org/10.1038/srep00910.

10. Chu, Y., Kompoliti, K., Cochran, E.J., Mufson, E.J. and Kordower, J.H., 2002. Age-related decreases in Nurr1 immunoreactivity in the human substantia nigra. The Journal of comparative neurology, 450(3): 203-14. https://doi.org/10.1002/cne.10261.

11. Chu, Y. and Kordower, J.H., 2007. Age-associated increases of alpha-synuclein in monkeys and humans are associated with nigrostriatal dopamine depletion: Is this the target for Parkinson's disease? Neurobiology of disease, 25(1): 134-49. https://doi.org/10.1016/j.nbd.2006.08.021.

12. Davidson, W.S., Jonas, A., Clayton, D.F. and George, J.M., 1998. Stabilization of alpha-synuclein secondary structure upon binding to synthetic membranes. J Biol Chem, 273(16): 9443-9. https://doi.org/10.1074/jbc.273.16.9443.

13. Forsyth, C.B. et al., 2011. Increased intestinal permeability correlates with sigmoid mucosa alphasynuclein staining and endotoxin exposure markers in early Parkinson's disease. PloS one, 6(12): e28032. https://doi.org/10.1371/journal.pone.0028032.

14. Frost, B. and Diamond, M.I., 2010. Prion-like mechanisms in neurodegenerative diseases. Nature reviews. Neuroscience, 11(3): 155-9. https://doi.org/10.1038/nrn2786.

15. Gelpi, E. et al., 2014. Multiple organ involvement by alpha-synuclein pathology in Lewy body disorders. Movement disorders : official journal of the Movement Disorder Society, 29(8): 1010-8. https://doi.org/10.1002/mds.25776.

16. Goedert, M., 2001. Alpha-synuclein and neurodegenerative diseases. Nature reviews. Neuroscience, 2(7): 492-501. https://doi.org/10.1038/35081564.

17. Gordon, R. et al., 2018. Inflammasome inhibition prevents a-synuclein pathology and dopaminergic neurodegeneration in mice. Science translational medicine, 10(465). https://doi.org/10.1126/scitransImed.aah4066.

18. Halliday, G.M. and McCann, H., 2008. Human-based studies on alpha-synuclein deposition and relationship to Parkinson's disease symptoms. Experimental neurology, 209(1): 12-21. 
https://doi.org/10.1016/j.expneurol.2007.07.006.

19. Hawkes, C.H., Del Tredici, K. and Braak, H., 2009. Parkinson's disease: the dual hit theory revisited. Ann N Y Acad Sci, 1170: 615-22. https://doi.org/10.1111/j.1749-6632.2009.04365.x.

20. Heneka, M.T., McManus, R.M. and Latz, E., 2018. Inflammasome signalling in brain function and neurodegenerative disease. Nature reviews. Neuroscience, 19(10): 610-621.

https://doi.org/10.1038/s41583-018-0055-7.

21. Holmqvist, S. et al., 2014a. Direct evidence of Parkinson pathology spread from the gastrointestinal tract to the brain in rats. Acta Neuropathol, 128(6): 805-20. https://doi.org/10.1007/s00401-014$1343-6$.

22. Holmqvist, S. et al., 2014b. Direct evidence of Parkinson pathology spread from the gastrointestinal tract to the brain in rats. Acta Neuropathol, 128(6): 805-20. https://doi.org/10.1007/s00401-0141343-6.

23. Houser, M.C. and Tansey, M.G., 2017. The gut-brain axis: is intestinal inflammation a silent driver of Parkinson's disease pathogenesis? NPJ Parkinsons Dis, 3: 3. https://doi.org/10.1038/s41531-0160002-0.

24. Jellinger, K.A., 2019. Is Braak staging valid for all types of Parkinson's disease? Journal of neural transmission, 126(4): 423-431. https://doi.org/10.1007/s00702-018-1898-9.

25. Kiely, A.P. et al., 2013. alpha-Synucleinopathy associated with G51D SNCA mutation: a link between Parkinson's disease and multiple system atrophy? Acta Neuropathol, 125(5): 753-69. https://doi.org/10.1007/s00401-013-1096-7.

26. Krainc, Dimitri, Wong, Yvette and C., 2017. alpha-synuclein toxicity in neurodegeneration: mechanism and therapeutic strategies. Nature Medicine.

27. Kruger, R. et al., 1998. Ala30Pro mutation in the gene encoding alpha-synuclein in Parkinson's disease. Nat Genet, 18(2): 106-8. https://doi.org/10.1038/ng0298-106.

28. Lesage, S. et al., 2013. G51D alpha-synuclein mutation causes a novel parkinsonian-pyramidal syndrome. Ann Neurol, 73(4): 459-71. https://doi.org/10.1002/ana.23894.

29. Li, W. et al., 2004. Stabilization of ??-synuclein protein with aging and familial Parkinson's diseaselinked A53T mutation. The Journal of neuroscience : the official journal of the Society for Neuroscience, 24: 7400-9. https://doi.org/10.1523/JNEUROSCI.1370-04.2004.

30. Li, W. et al., 2005. Aggregation promoting C-terminal truncation of alpha-synuclein is a normal cellular process and is enhanced by the familial Parkinson's disease-linked mutations. Proceedings of the National Academy of Sciences of the United States of America, 102(6): 2162-7. https://doi.org/10.1073/pnas.0406976102.

31. Ltic, S. et al., 2004. Alpha-synuclein is expressed in different tissues during human fetal development. Journal of molecular neuroscience : MN, 22(3): 199-204. https://doi.org/10.1385/jmn:22:3:199.

32. Mak, S., McCormack, A., Langston, J., Kordower, J. and Di Monte, D., 2009a. Decreased a-synuclein expression in the aging mouse substantia nigra. Experimental neurology, 220: 359-65. 
https://doi.org/10.1016/j.expneurol.2009.09.021.

33. Mak, S.K., McCormack, A.L., Langston, J.W., Kordower, J.H. and Di Monte, D.A., 2009b. Decreased alpha-synuclein expression in the aging mouse substantia nigra. Experimental neurology, 220(2): 359-65. https://doi.org/10.1016/j.expneurol.2009.09.021.

34. Morens, D.M. et al., 1996. Epidemiologic observations on Parkinson's disease: incidence and mortality in a prospective study of middle-aged men. Neurology, 46(4): 1044-50. https://doi.org/10.1212/wnl.46.4.1044.

35. Nuber, S. and Selkoe, D.J., 2016. Caspase-1 clipping causes complications for alpha-synuclein. Proceedings of the National Academy of Sciences of the United States of America, 113(36): 9958-60. https://doi.org/10.1073/pnas.1610993113.

36. Oaks, A.W., Frankfurt, M., Finkelstein, D.I. and Sidhu, A., 2013. Age-dependent effects of A53T alphasynuclein on behavior and dopaminergic function. PloS one, 8(4): e60378. https://doi.org/10.1371/journal.pone.0060378.

37. Orimo, S. et al., 2008. Axonal alpha-synuclein aggregates herald centripetal degeneration of cardiac sympathetic nerve in Parkinson's disease. Brain, 113: 81-86.

38. Pasanen, P. et al., 2014. Novel alpha-synuclein mutation A53E associated with atypical multiple system atrophy and Parkinson's disease-type pathology. Neurobiol Aging, 35(9): 2180 e1-5. https://doi.org/10.1016/j.neurobiolaging.2014.03.024.

39. Phillips, R.J., Walter, G.C., Ringer, B.E., Higgs, K.M. and Powley, T.L., 2009. Alpha-synuclein immunopositive aggregates in the myenteric plexus of the aging Fischer 344 rat. Experimental neurology, 220(1): 109-19. https://doi.org/10.1016/j.expneurol.2009.07.025.

40. Polymeropoulos, M.H. et al., 1997. Mutation in the alpha-synuclein gene identified in families with Parkinson's disease. Science, 276(5321): 2045-2047.

41. Pouclet, H. et al., 2012. A comparison between rectal and colonic biopsies to detect Lewy pathology in Parkinson's disease. Neurobiology of disease, 45(1): 305-9. https://doi.org/10.1016/j.nbd.2011.08.014.

42. Proukakis, C. et al., 2013. A novel alpha-synuclein missense mutation in Parkinson disease. Neurology, 80(11): 1062-4. https://doi.org/10.1212/WNL.0b013e31828727ba.

43. Ross, G.W. et al., 2004. Parkinsonian signs and substantia nigra neuron density in decendents elders without PD. Ann Neurol, 56(4): 532-9. https://doi.org/10.1002/ana.20226.

44. Rudow, G. et al., 2008. Morphometry of the human substantia nigra in ageing and Parkinson's disease. Acta Neuropathol, 115(4): 461-70. https://doi.org/10.1007/s00401-008-0352-8.

45. Shprecher, D.R. and Derkinderen, P., 2012. Parkinson disease: the enteric nervous system spills its guts. Neurology, 78(9): 683, author reply 683. https://doi.org/10.1212/WNL.0b013e31824bd195.

46. Silva, B.A., Breydo, L. and Uversky, V.N., 2013. Targeting the chameleon: a focused look at alphasynuclein and its roles in neurodegeneration. Mol Neurobiol, 47(2): 446-59. https://doi.org/10.1007/s12035-012-8334-1. 
47. Tschopp, J. and Schroder, K., 2010. NLRP3 inflammasome activation: The convergence of multiple signalling pathways on ROS production? Nature reviews. Immunology, 10(3): 210-5. https://doi.org/10.1038/nri2725.

48. Ulusoy, A., Febbraro, F., Jensen, P.H., Kirik, D. and Romero-Ramos, M., 2010. Co-expression of Cterminal truncated alpha-synuclein enhances full-length alpha-synuclein-induced pathology. European Journal of Neuroscience, 32(3): 409-422. https://doi.org/10.1111/j.14609568.2010.07284.x.

49. Uversky, V.N., 2017. Looking at the recent advances in understanding alpha-synuclein and its aggregation through the proteoform prism. F1000Research, 6: 525. https://doi.org/10.12688/f1000research.10536.1.

50. Wang, W. et al., 2016. Caspase-1 causes truncation and aggregation of the Parkinson's diseaseassociated protein a-synuclein. Proceedings of the National Academy of Sciences of the United States of America, 113(34): 9587-92. https://doi.org/10.1073/pnas.1610099113.

51. Zarranz, J.J. et al., 2004. The new mutation, E46K, of alpha-synuclein causes Parkinson and Lewy body dementia. Ann Neurol, 55(2): 164-73. https://doi.org/10.1002/ana.10795.

52. Zhong, C.B. et al., 2017. Age-Dependent Alpha-Synuclein Accumulation and Phosphorylation in the Enteric Nervous System in a Transgenic Mouse Model of Parkinson's Disease. Neuroscience bulletin, 33(5): 483-492. https://doi.org/10.1007/s12264-017-0179-1.

\section{Figures}




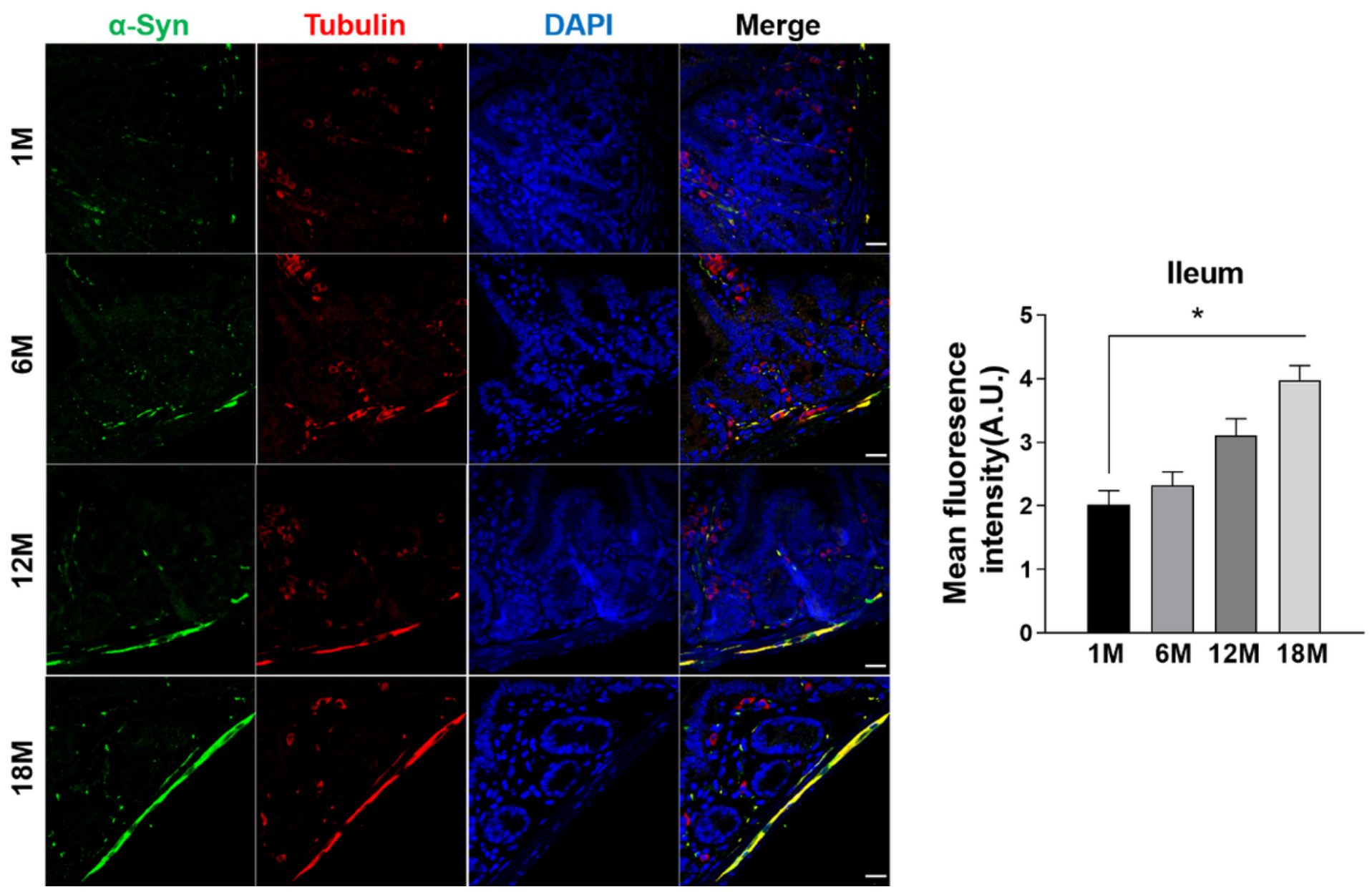

Figure 1

Ileum was stained with antibodies against a-Syn (green), Tubulin (red), and DAPI (blue) .Scale bar $=20$ $\mu \mathrm{m}$. Data are presented as means $\pm \mathrm{SD}$. ${ }^{\star} \mathrm{p}<0.05$ compared with the 1 -month-old group. 

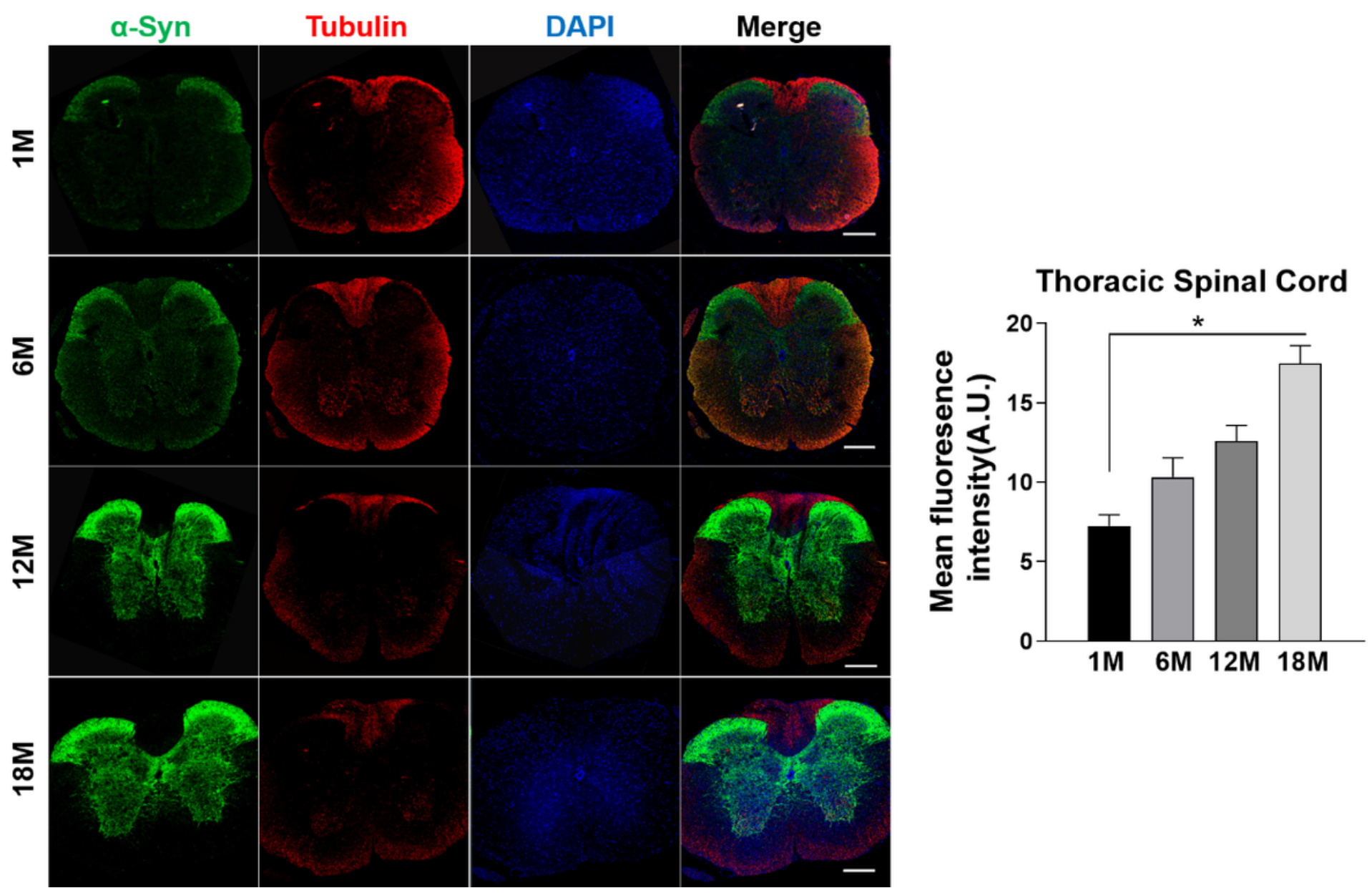

Figure 2

Fluorescence of mouse thoracic spinal cord in different ages. a-Syn (green), Tubulin (red), and DAPI (blue). Scale bar $=200 \mu \mathrm{m}$. Data are presented as means \pm SD. ${ }^{*} p<0.05$ compared with the 1 -month-old group. 

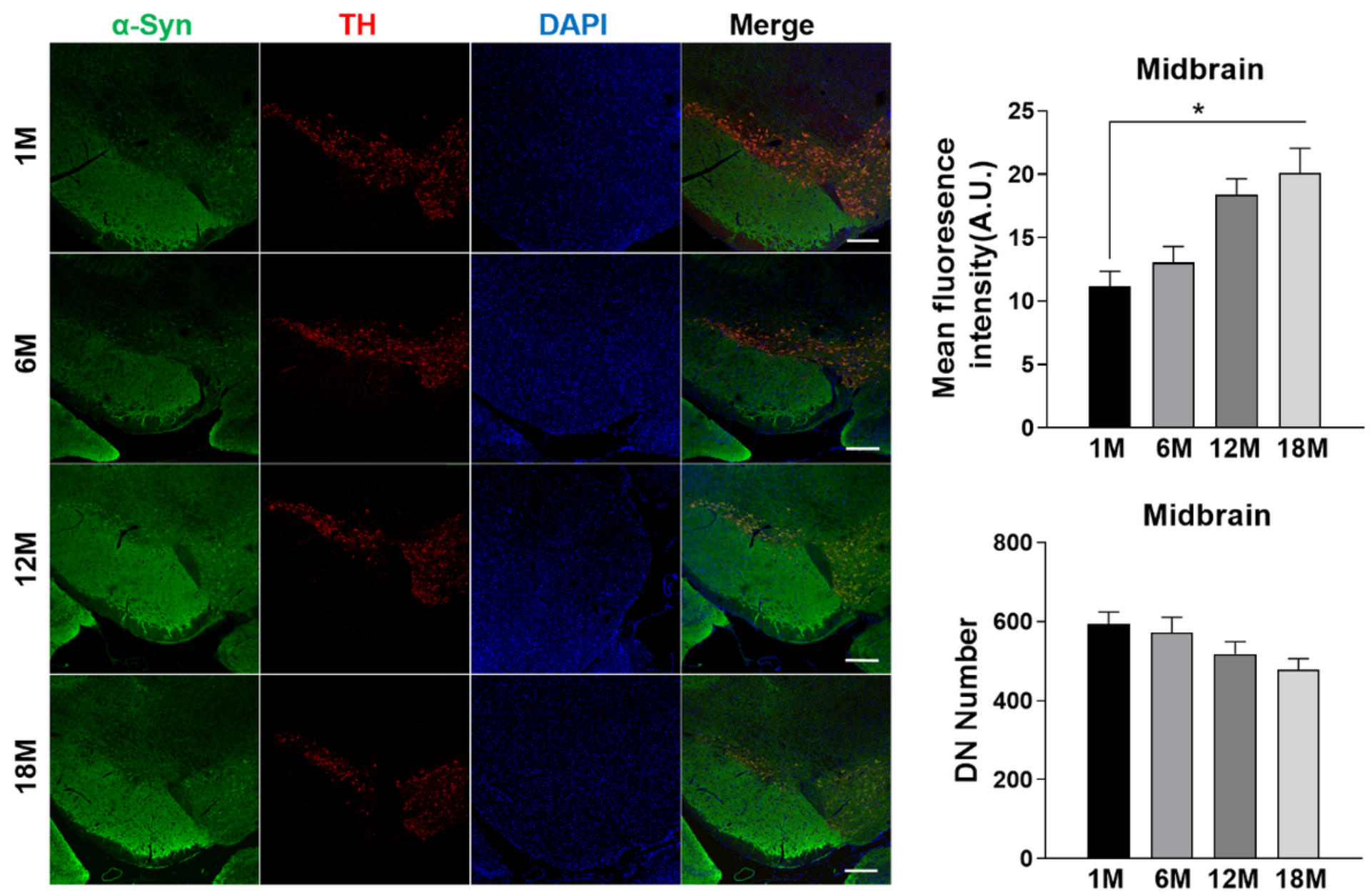

Figure 3

Fluorescence staining of a-Syn (green), TH (red), and DAPI (blue) in the midbrain of 1-, 6-, 12-, and 18month-old mice. Scale bar $=200 \mu \mathrm{m}$. Data are presented as means \pm SD. ${ }^{*} p<0.05$ compared with the 1month-old group. 
A
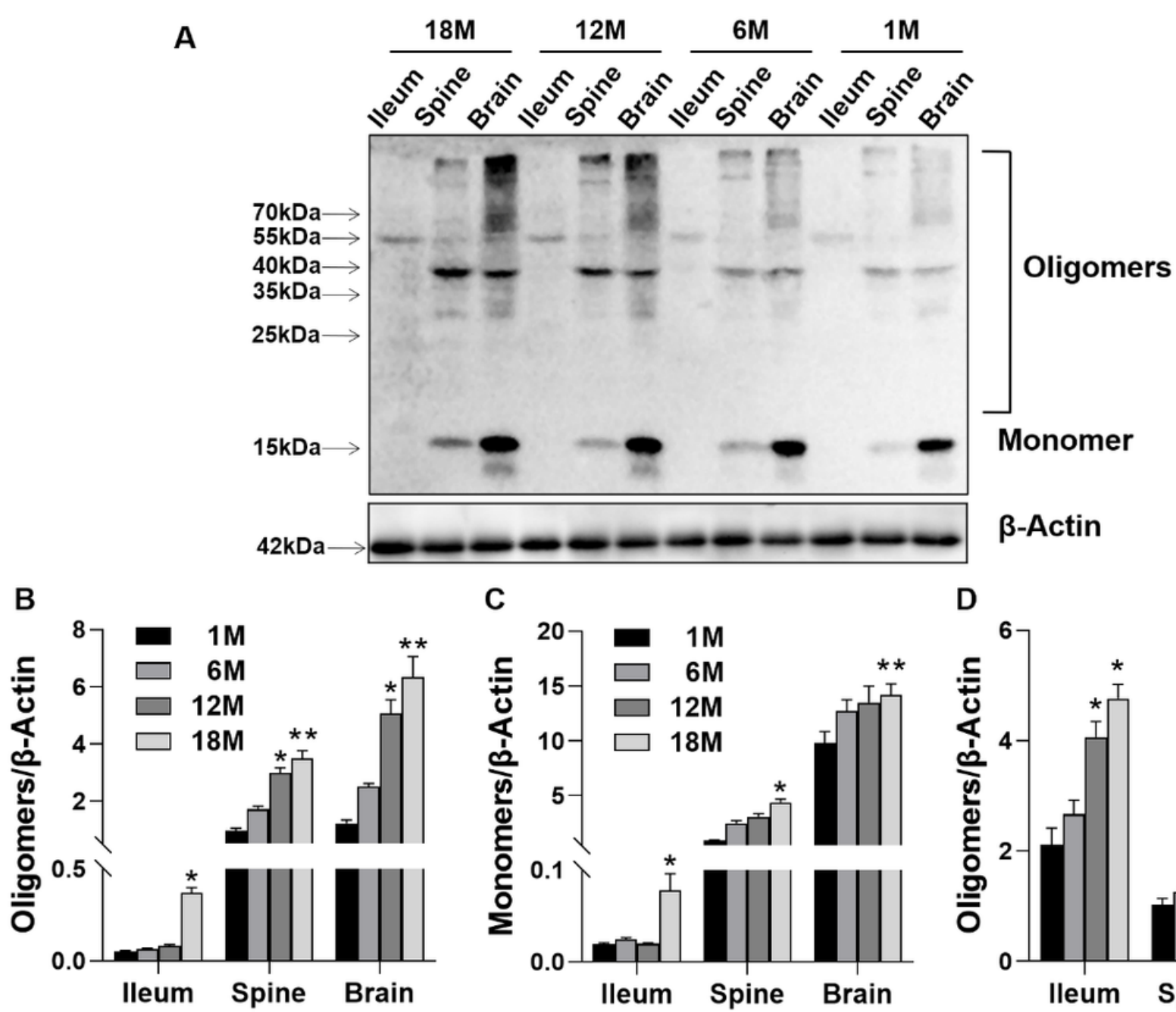

C
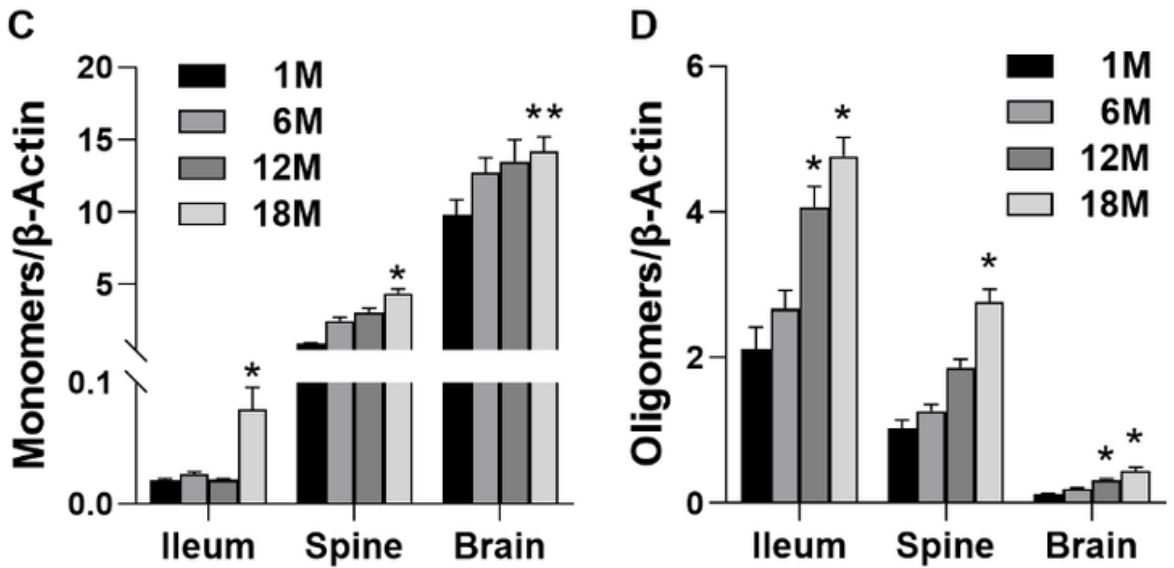

Figure 4

Western blotting analysis for oligomers and monomer a-Syn in the ileum, thoracic spinal cord and midbrain of different month-old mice. $\beta$-Actin was used as a reference control. Data are presented as means \pm SD. $n=5$ per group. ${ }^{*} p<0.05$ compared with the 1-month-old group. 


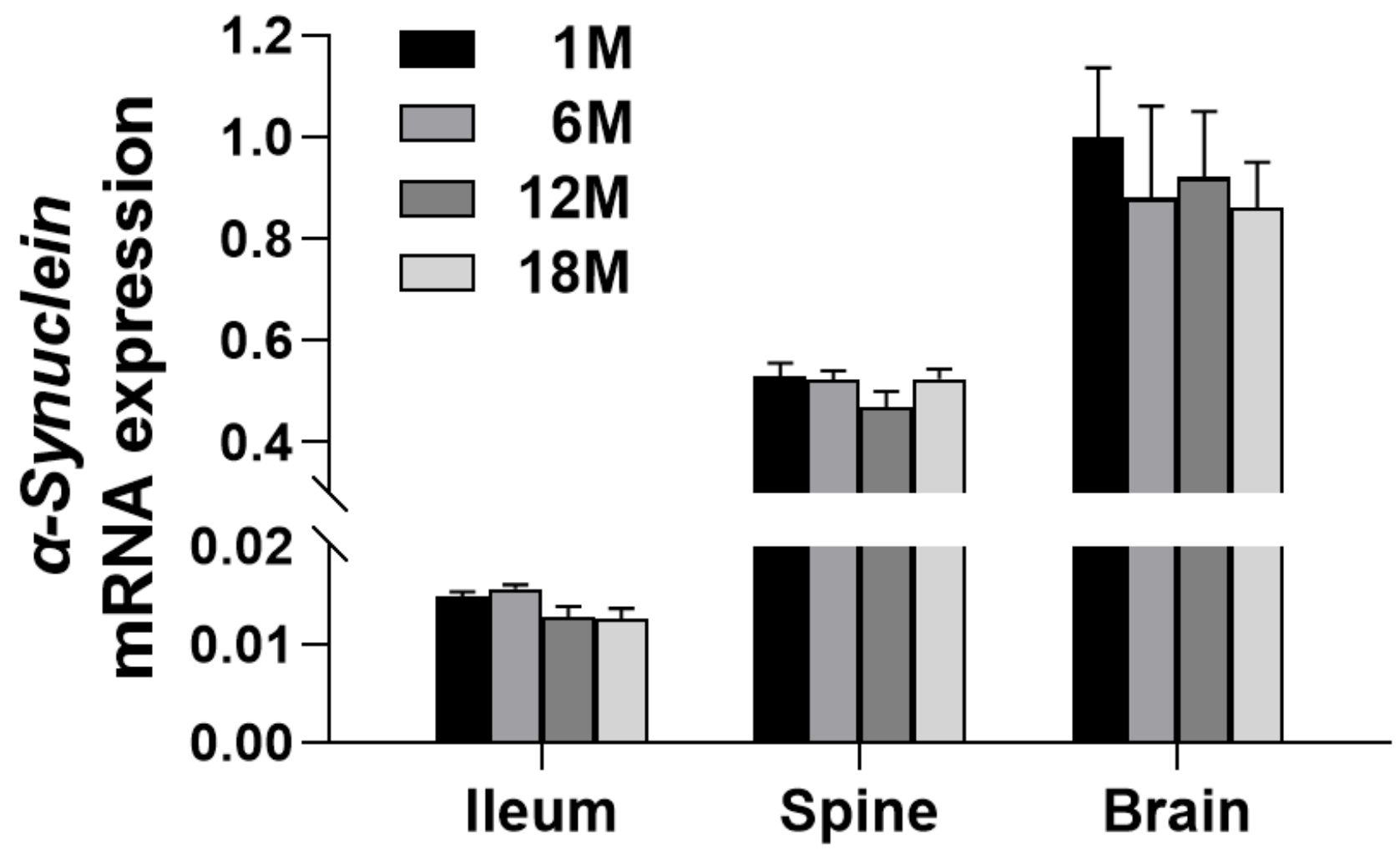

Figure 5

Expression levels of a-Syn mRNA in samples from the ileum, spinal cord and brain of mice at 1, 6, 12, and 18-month by real-time PCR. The data are shown as means \pm SD. $n=8$ per group. 

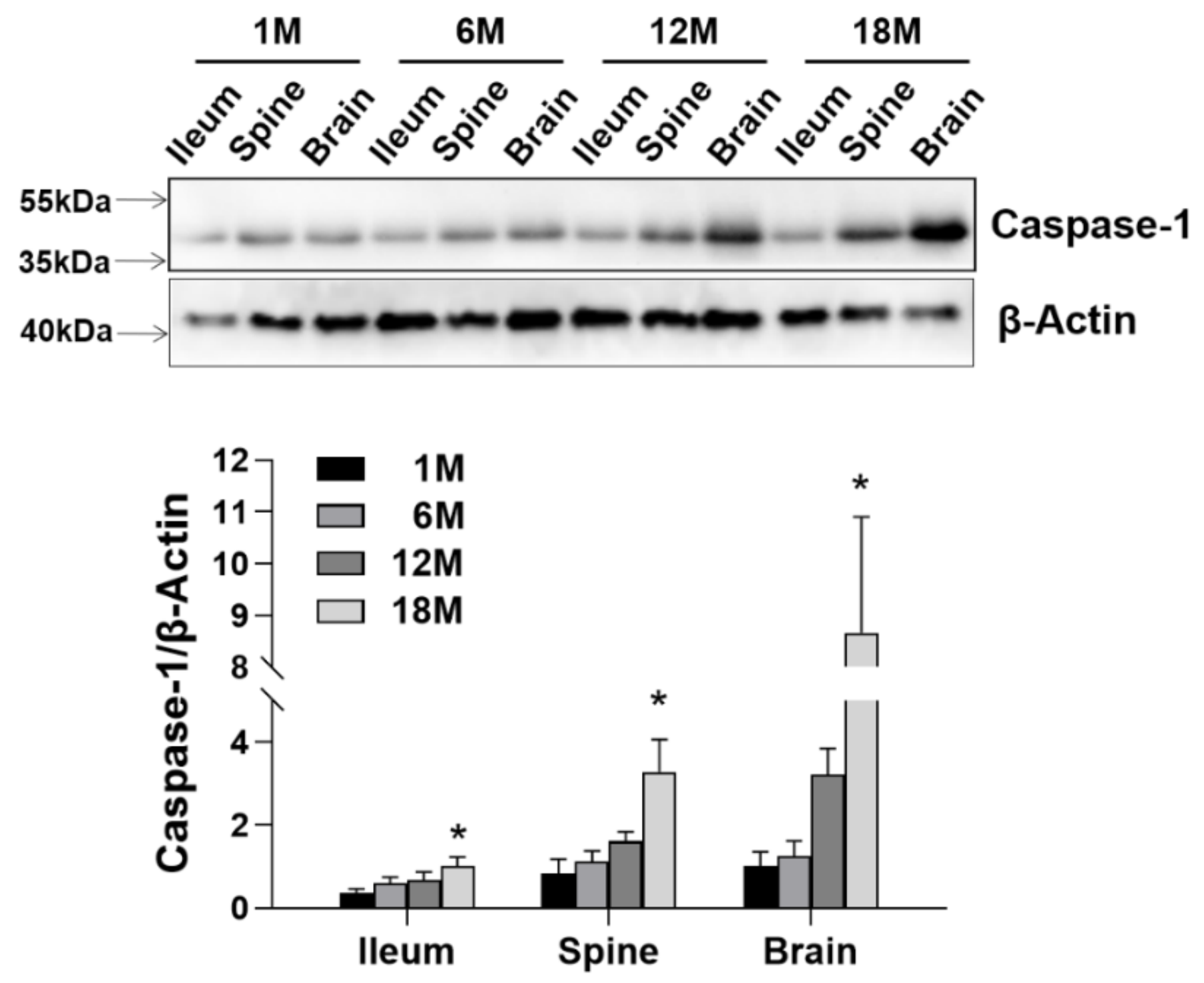

Figure 6

The expression of caspase-1 protein in the different month-old mouse ileum, thoracic spinal cord and midbrain. $\beta$-Actin used as a reference control. Data are presented as means \pm SD. $n=5$ per group. ${ }^{*} p<0.05$ compared with the 1-month-old group. 

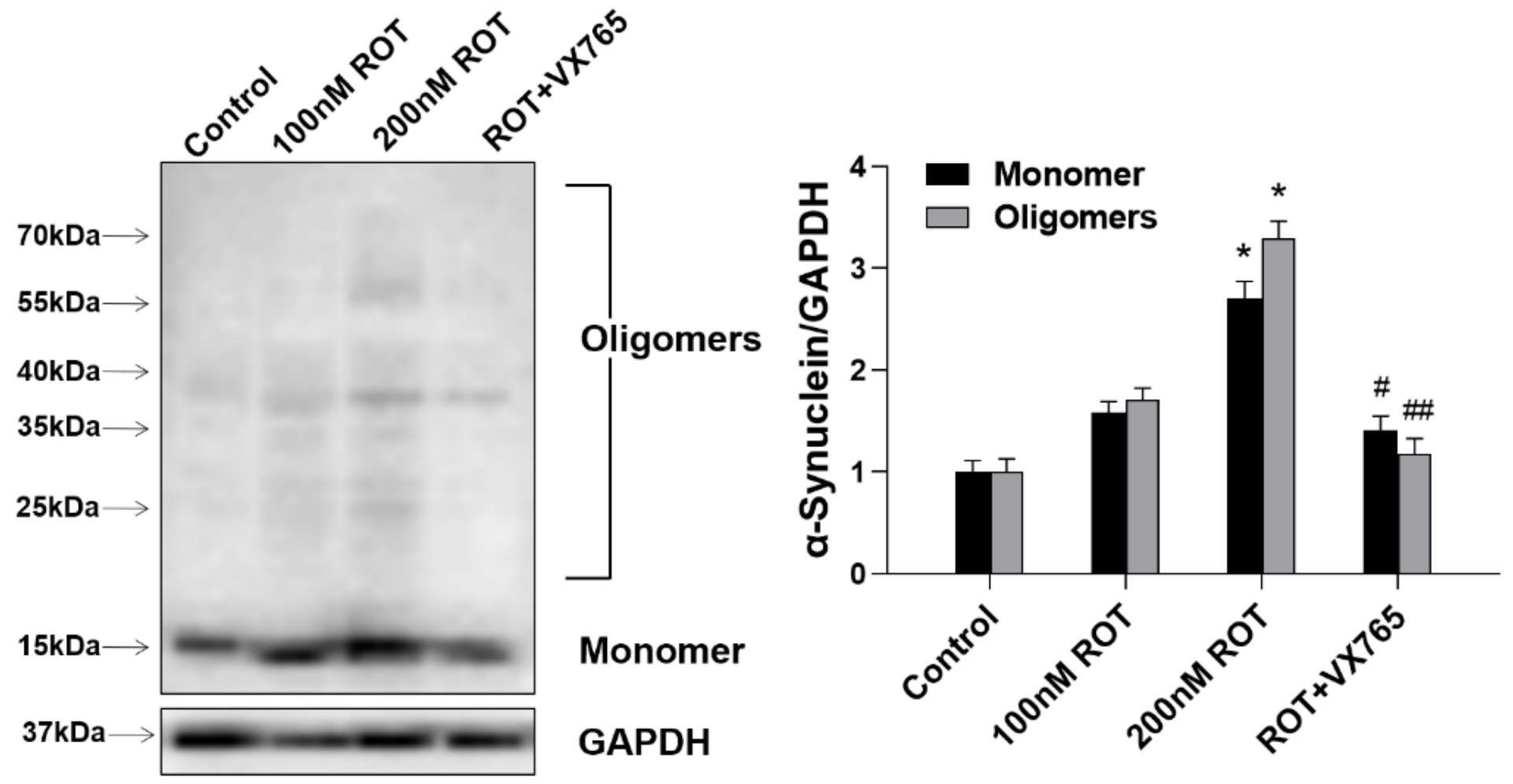

Figure 7

SH-SY5Y cells were treated with PBS, 100 nM ROT, 200 nM ROT, $200 \mathrm{nM} \mathrm{ROT+VX765.} \mathrm{Western} \mathrm{blotting}$ analysis for a-Syn monomers and oligomers. GAPDH was used as a reference control. $\mathrm{n}=3$ per group. ${ }^{\star} p<0.05,{ }^{\star \star} p<0.01$ compared with control group. $\# p<0.05, \# \# p<0.01$ compared with the $200 \mathrm{nM}$ ROT group. 


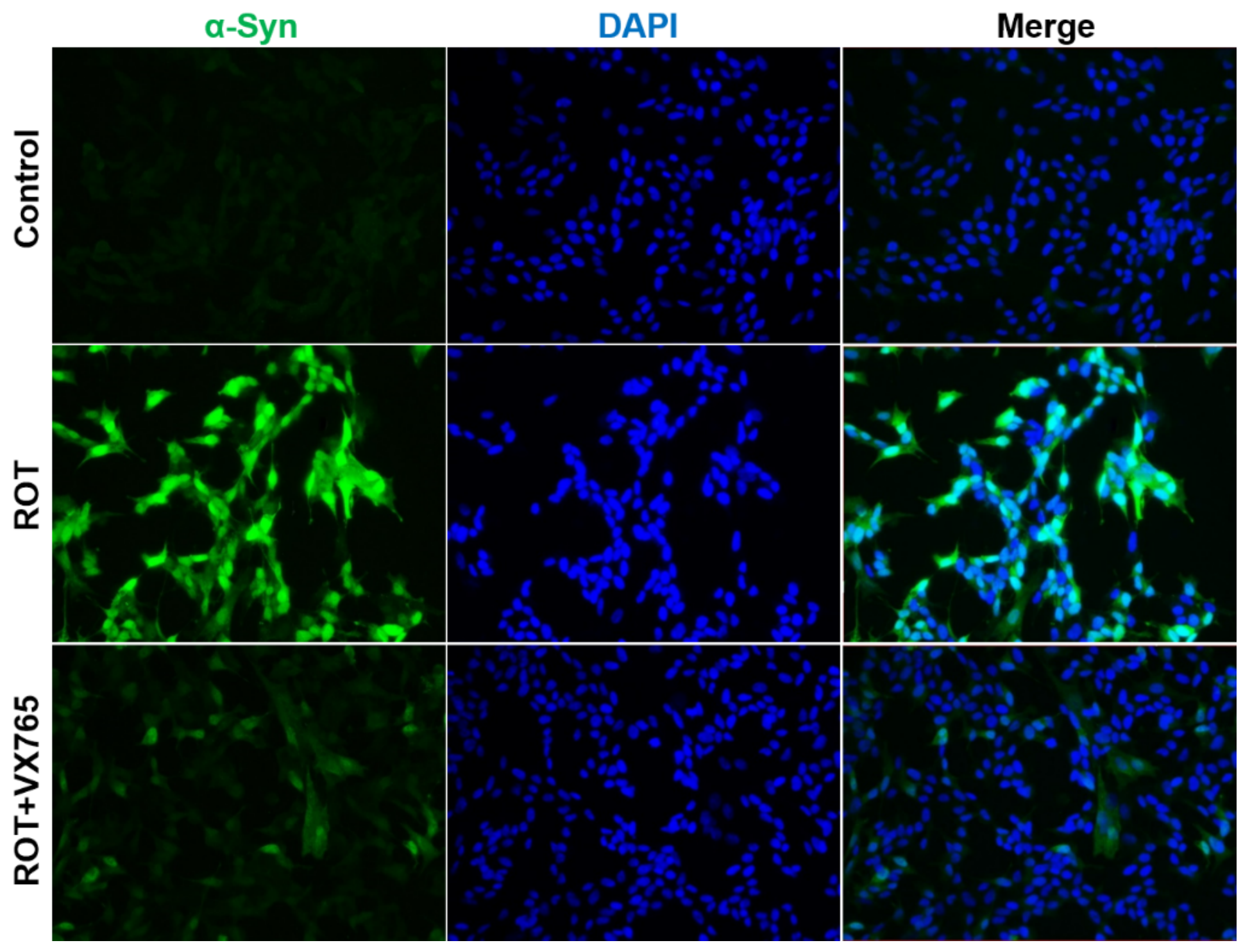

Figure 8

Fluorescence images of a-Syn (green) and DAPI (blue) in SH-SY5Y cells treated with PBS,200 nM ROT, $200 \mathrm{nM}$ ROT+VX765. 